\title{
Germanica
}

36 | 2005

Le pouvoir de la musique dans l'espace de langue allemande : fascination et suspicion

\section{« Eine Magierin in entzauberter Welt ». Le pouvoir de la musique dans Schlafes Bruder de Robert Schneider}

"Eine Magierin in entzauberter Welt »-Le pouvoir de la musique dans Schlafes

Bruder de Robert Schneider

Jean-Charles Margotton

\section{(2) OpenEdition}

Journals

Édition électronique

URL : http://journals.openedition.org/germanica/1530

DOI : 10.4000/germanica. 1530

ISSN : 2107-0784

Éditeur

Université de Lille

Édition imprimée

Date de publication : 30 juin 2005

Pagination : 181-193

ISBN : 9782913857155

ISSN : 0984-2632

Référence électronique

Jean-Charles Margotton, « «Eine Magierin in entzauberter Welt ». Le pouvoir de la musique dans Schlafes Bruder de Robert Schneider », Germanica [En ligne], 36 | 2005, mis en ligne le 16 juillet 2012, consulté le 06 octobre 2020. URL : http://journals.openedition.org/germanica/1530 ; DOI : https:// doi.org/10.4000/germanica.1530

Ce document a été généré automatiquement le 6 octobre 2020.

(c) Tous droits réservés 


\title{
« Eine Magierin in entzauberter Welt $»^{1}$. Le pouvoir de la musique dans Schlafes Bruder de Robert Schneider
}

\author{
"Eine Magierin in entzauberter Welt »-Le pouvoir de la musique dans Schlafes \\ Bruder de Robert Schneider
}

Jean-Charles Margotton

1 Les réactions au dernier roman de Robert Schneider, Kristus², ont été mitigées et parmi elles, il y a eu à nouveau de violentes critiques dirigées contre des textes que certains voudraient rabaisser au nouveau de la littérature de gare. Même son premier roman, Schlafes Bruder, qui lui a pourtant valu d'emblée une grande célébrité n'a pas échappé à ce genre de critique. Mais nous n'entrerons pas dans le débat touchant la qualité littéraire d'une œuvre dont ne nous séparent encore que deux décennies à peine. Admettons que nous manquons de recul et que le succès subit et international du roman, peut-être confirmé, en tout cas répercuté par sa transcription au cinéma et à l'opéra, n'est pas forcément gage de valeur et de succès pérenne.

2 Que la musique soit un thème central de ce roman et qu'on retrouve ce thème ensuite dans les autres parties de sa "trilogie du val rhénan $»^{3}$ ne peut surprendre s'agissant d'un écrivain qui a étudié la musique en même temps que l'art dramatique et l'histoire de l'art à Vienne et qui a travaillé comme organiste avant de devenir un écrivain vivant de sa plume. De même que l'univers où est située l'histoire est le pays montagnard du Vorarlberg où est né et où vit encore l'auteur, de même il est évident que les pages du roman consacrées à l'orgue, à sa technique, aux particularités de son jeu, à l'écriture classique de la musique d'église supposent un écrivain au fait de la question, et pas seulement un amateur. Dans le roman Die Unberührten, c'est - et là aussi au milieu d'un foisonnement complexe d'épisodes et de situations contrastées - le monde de l'opéra qui est thématisé. Le héros du premier roman a ici, un siècle plus tard, un équivalent 
féminin, la petite Antonia, qui manifeste dans le domaine du chant lyrique la même disposition géniale spontanée qu'Elias, le héros de Schlafes Bruder, à l'orgue.

$3 \mathrm{Au}$ demeurant, la musique est un thème assez fréquent dans la littérature germanophone récente, $\mathrm{R}$. Schneider n'est pas en cela vraiment un cas isolé. On peut songer à E. Jelinek (Die Klavierspielerin), à Th. Bernhard (Der Untergeher), à P. Süskind (Der Kontrabass), à H. J. Ortheil (Die Nacht des Don Juan), H. Krausser (Melodien) et à bien d'autres. Il n'est donc pas question d'étudier ici un phénomène unique, mais de voir en quoi le thème est ici traité de manière originale et comment il est intégré à la matière du roman dans son ensemble.

4 Cette place de la musique dans une narration renvoie bien sûr aussi, en particulier s'agissant de littérature germanophone, à une tradition déjà longue et il conviendra de faire certains rapprochements éclairants entre le roman contemporain et la tradition romantique, plus précisément avec Wackenroder et Hoffmann. La fiction de R. Schneider n'est sans doute pas par hasard située dans les premières décennies du dix-neuvième siècle et si la musique d'orgue que le naiff et malheureux petit paysan, Elias, découvre presque seul et pratique de manière géniale, s'inscrit clairement dans la tradition de Bach, le discours métaphysique sur son art et sur son destin évoque trop souvent et trop précisément les textes programmatiques des Romantiques pour qu'il y ait un effet du hasard. Évidemment, le roman postmoderne se plait à mêler genres et traditions, mais nous tenterons de montrer que cette référence romantique constitue un élément déterminant de la construction complexe de cette œuvre attachante et déroutante à la fois.

5 La musique d'ailleurs, il faut bien le voir, n'est pas le seul thème du roman malgré son importance. Elle fait plutôt partie d'une connexion dont le héros est le centre. Les premiers chapitres retardent le commencement de l'histoire d'Elias en évoquant les catastrophes qui ont fini par détruire son village après sa mort et en chasser tous les habitants. Le roman commence ainsi par la fin de l'histoire et même par l'épilogue qui, d'ailleurs, sera repris dans les deux derniers chapitres. L'histoire centrale ne débute vraiment qu'au chapitre 4 . Mais le cadre thématique et le sens du texte auront été d'emblée formulés par le narrateur qui, sur un ton solennel et accusateur, présente l'histoire d'Elias comme celle d'un de ces génies restés totalement inconnus alors qu'ils ont peut-être été supérieurs à ceux qui ont façonnés le monde :

Elle n'est autre qu'un réquisitoire contre Dieu, qui, dans ses caprices de dilapidateur, avait jugé bon de répandre justement le talent si précieux de la musique sur un enfant de paysans d'Eschberg quand il aurait dû prévoir que celui-ci ne pourrait jamais, dans cette contrée si étrangère aux muses, mettre à profit ces dons ni les parachever.4. (FS, p. 13)

6 C'est comme si ces êtres n'étaient même pas nés, comme le suggère le titre du troisième chapitre. Les nombreuses références chrétiennes, comme déjà le titre du roman qui se révélera être une citation d'un choral luthérien, ne font que renforcer l'absurdité, l'injustice, la violence, en un mot la négativité du monde représenté. C'est dans ce cadre que doit être vu le thème de la musique, comme une composante d'un monde traversé de contradictions que le narrateur, malgré sa position supérieure, ne peut ni ne veut résoudre. Aussi bien la mort du héros n'est-elle pas la simple conséquence de l'inadéquation entre son génie et le milieu misérable et barbare où l'a jeté la naissance, elle est provoquée par le héros lui-même, amoureux d'Elsbeth, jeune paysanne en qui, avant même qu'elle naisse, il voit sa destinée, mais qui ne comprend pas son amour et en est effrayé. Ainsi musique et amour sont liés comme deux manifestations d'une 
même force proprement surhumaine, venue habiter un être pour le façonner, le transformer, l'élever et, finalement, l'écraser.

7 C'est donc un phénomène complexe et contradictoire que nous analysons. D'une part, et nous commencerons par cet aspect, le pouvoir de la musique est manifesté par la manière dont le génie du musicien naïf est mis en rapport avec une musique universelle, avec la musique du monde, puis par l'influence extraordinaire qu'il exerce sur les êtres qui l'entourent; d'autre part, il conviendra d'analyser les éléments qui font du prodige un être étrange, un monstre qui finit par se détruire lui-même, écrasé à la fois par le poids de la réalité et par la force inhumaine qui l'habite.

Un aspect sensationnel du roman est la description de la transformation que subit l'enfant Elias lorsque, seul, loin de son village montagnard et près de la pierre étrange sur laquelle il mourra, il est investi violemment par tous les bruits de l'univers qu'il reçoit comme s'il s'agissait d'un concert :

C'est alors que vint le miracle. Cet après-midi-là, Elias entendit résonner l'univers ${ }^{5}$.

(FS, p. 33)

9 Le génie de la musique vient en lui d'abord sous la forme d'une ouie surhumaine puisqu'il fait la découverte de ce "miracle » en entendant tomber la neige alors qu'il est enfermé depuis de nombreux jours dans sa chambre. Puis, au terme d'une métamorphose violente dont tout son corps est l'objet, il devient capable d'imiter les voix des animaux et ainsi de les charmer, comme un second Orphée. La découverte de la musique des hommes se fait de manière également miraculeuse. Déjà la naissance du héros n'a pu se réaliser qu'à partir du moment où la sage-femme, voyant que l'enfant restait inerte après l'accouchement, s'était mise à chanter le Te deum. Le petit être, mis au monde au terme de longues souffrances, refuse de naître avant d'entendre la première mélodie de son existence. Ce Te deum qui lui sauve la vie est la première marque du pouvoir de la musique. En réalité, rien de prédispose l'enfant à un destin de musicien. La seule présence musicale dans ce village déshérité est celle de l'orgue de la petite église, un instrument sur lequel Oskar, un oncle d'Elias, joue pendant les offices. Le jeune prodige apprend non seulement seul à jouer, mais il découvre rapidement les défauts du misérable instrument et parvient à le réparer en secret, si bien que l'organiste titulaire s'en évanouit de saisissement quand il se met à jouer le lendemain. L'apprentissage de la musique se fait de manière spontanée, volontaire et miraculeuse à la fois, Elias parvient, sans nulle méthode, sans l'aide de quiconque et malgré l'extrême brutalité de son monde, à comprendre, puis à interpréter l'écriture contrapuntique, et même à en renouveler les structures. Son génie met en rapport, sans heurt, comme naturellement, l'harmonie de la Nature et la musique d'église de la tradition de Bach dont il comprend d'emblée les structures complexes. Cette étrange carrière du musicien prodige culmine lors du concours de jeunes talents auquel Elias est convié à participer dans la ville proche : la manière dont il interprète et ornemente le choral "Komm, o Tod, du Schlafes Bruder » bouleverse tout ce que les spécialistes présents ont entendu en matière de contrepoint et de figuralisme. Son jeu, que le narrateur est capable de décrire à l'aide d'un langage savant et de termes techniques, est apparemment totalement intuitif: la musique qu'il joue est celle qu'il compose, en improvisant sur les voix ou les images du monde. C'est le rythme des battements du cœur d'Elsbeth dont il a découvert qu'il était en isorythmie parfaite avec le sien; ou bien c'est le vol de deux papillons qui lui suggère l'invention d'une polyphonie à deux voix : 
Un jour qu'il rêvait, allongé dans l'herbe, il observait les évolutions de deux citrins qui pirouettaient joyeusement de-ci, de-là. C'est ainsi qu'il commença à ajouter une nouvelles mélodie à l'ancienne ${ }^{6}$. (FS, p. 66)

10 Ce génial musicien, habité par la musique sans la connaître, sans savoir lire une note, n'est pas seulement transporté lui-même par les beautés qu'il fait naître sous ses doigts, sa brève existence est aussi marquée par les moments où s'est manifesté sur les autres autour de lui le pouvoir étrange de la musique. À la manière de Grenouille, le héros de Süskind, qui parvient à composer un parfum au pouvoir de séduction absolu et diabolique, Elias trouve dans la voix qu'il s'est forgée et dans la maîtrise surhumaine de son instrument la capacité de subjuguer les hommes :

Il avait hypnotisé l'assistance. Les auditeurs étaient immobiles sur leurs bancs, leurs paupières ne bougeaient plus. Leur respiration s'était ralentie, et la fréquence des battements de leur cœur était celle de son propre cœur. [...] Au fil de l'inépuisable combinaison des accords naissent des configurations dont la sonorité déclenche chez l'auditeur quelque chose qui, en fait, n'a plus rien à voir avec la musique ${ }^{7}$. (FS, pp. 165 sq.)

11 À cet égard, le centre du roman correspond à la fête de Pâques, au cours de laquelle Elias peut enfin prendre la place de l'organiste Oskar, mort d'avoir pris conscience de l'insuffisance de son talent et du génie de son neveu. Le passage est un véritable morceau de bravoure :

Les fidèles furent saisis d'un puissant étonnement quand soudain, au Gloria, l'orgue se mit à gronder, manifestant par l'allégresse du doigté comment un chrétien devait se réjouir de cette commémoration. Elias donna une toccata pleine d'ampleur qui se terminait par un fugato sur la mélodie du cantique ${ }^{8}$. (FS, p. 108)

Cette musique inédite dépasse tout ce que les pauvres villageois ont jamais pu entendre, mais leur étonnement n'est pas le seul trouble qu'elle provoque en eux ; pour un instant, ils s'en trouvent transformés et comme ennoblis :

La musique de l'organiste avait rendu la piété et la douceur de l'agneau à leurs cœurs endurcis, car, singulièrement, personne ne partit avant la fin. Et l'on ne se bouscula pas non plus près du bénitier. Quelques-uns se mirent tout à coup à prendre des manières distinguées qui leur étaient tout à fait inhabituelles, cédaient le passage avec un geste élégant de leur main boudinée, et mêlaient à leurs salutations - on ne voudra pas le croire - des mots qui ressemblaient à du français ${ }^{9}$. (FS, p. 109)

13 Un autre morceau de bravoure reprend en l'exagérant le motif du pouvoir de la musique sur les auditeurs. Il s'agit, au $16^{\mathrm{e}}$ chapitre du roman, du concours d'orgue auquel Elias participe à Feldberg. Ses concurrents sont de jeunes citadins qui regardent de haut ce personnage aux pieds nus, descendu de sa montagne et qui ne sait pas même lire les notes. Il faut qu'on lui joue la mélodie du choral « Komm, o Tod, du Schlafes Bruder " sur lequel il doit improviser des variations. Mais à peine ce «curieux génie de la nature » (FS, p. 157) a-t-il commencé de jouer que tous, jeunes musiciens, membres du clergé et public dans la cathédrale, comprennent que ce qu'ils entendent est unique et dépasse toute imagination. De longues pages sont consacrées à la description de cette façon « inhumaine » d'interpréter la musique d'église. C'est qu'Elias, qui, pourtant, n'a entendu que les piètres réalisations musicales de son oncle, fait exploser les structures classiques de la musique liturgique. Son langage musical est si violent qu'aucun auditeur ne peut se soustraire à son pouvoir. Le narrateur décrit, en usant ponctuellement de termes techniques, ce concert qui semble ne pas vouloir finir et qui, bien plus, est comme une infinie gradation vers des hauteurs de plus en plus 
vertigineuses. Celui qui joue là exprime en effet, dans ce qui sera son ultime prestation, la totalité de ce qui constitue son univers, tous les paysages qu'il a vus, tous les êtres qu'il a connus et, avant tout, son amour pour Elsbeth ; son jeu devient même une sorte de prédication ( Non, l'homme qui était là-haut ne se contentait pas de faire de la musique, il faisait un sermon ${\aleph^{10}}$, FS, p. 163). Il parvient à faire sentir, dans son improvisation sur le choral, la présence glaçante de la mort, mais aussi la révolte de l'homme contre cette mort, contre son destin, contre Dieu. Enfin, lorsque virtuosité, complexité du contrepoint et intensité sonore semblent atteindre un point culminant, les auditeurs ont le sentiment d'être arrachés à leur monde terrestre et de «voir le ciel » (FS, p. 177). Elias parvient encore à dramatiser son jeu dans les derniers moments en interrompant brutalement un accord fortissimo, avant de redonner, non sans ornementation savante, la mélodie simple du choral.

Il semble difficile d'imaginer une image plus forte du pouvoir de la musique que celle qui se dégage de ces pages entièrement marquées par l'excès. Et le narrateur, au demeurant, ne se contente pas de marquer son admiration pour l'art surhumain de son héros, il ne masque pas ce que cet art a d'inquiétant et d'inhumain. Son jeu est « une improvisation inconcevable et, pour tout dire, folle » (FS, p. 179), la musique n'est pas en son pouvoir, même s'il éprouve en jouant une jubilation de plus en plus effrayante, le musicien est bien plutôt livré au pouvoir de la musique et quand enfin s'achève cette paroxystique prestation, elle rejette dans le monde un être dont elle a attaqué la substance :

Son visage déjà maigre avait la couleur de la cendre, ses joues étaient creusées, ses pommettes saillaient et ses lèvres étaient sèches ${ }^{11}$. (FS, p. 169)

Se répète ainsi d'une certaine façon la métamorphose initiale par laquelle Elias était devenu, enfant, un être à part, génie de l'ouïe et de la musique et monstre tout aussi bien.

Le thème de la musique, en effet, n'est pas épuisé par la représentation du génie spontané qui parvient à subjuguer ses auditeurs. La musique a une image ambivalente et participe de l'ambivalence générale d'un texte qui conjugue génie et monstruosité. La composition du roman fait alterner ainsi les passages consacrés à l'évolution du héros, depuis sa naissance inhabituelle, sa métamorphose et son autoformation à la musique jusqu'à son suicide, et, d'autre part, ceux qui décrivent le monde où il vit et les êtres qu'il côtoie. Or, c'est l'idée de barbarie, de violence, de stupidité et de primitivité qui se dégage de l'image du village, un monde plus inhumain qu'humain. Ainsi, pour prendre un exemple, l'ami unique d'Elias, le jeune Peter, est décrit à la fois comme une malheureuse victime de la brutalité de son père et comme un être capable lui-même de la pire cruauté. Son amitié amoureuse pour Elias, son admiration éperdue pour la musique dont il n'est capable de saisir que le pouvoir qu'elle exerce sur les autres, cela, certes, le fait sortir de lui-même parfois et peut-être est-il, pour finir, l'objet d'une espèce de rédemption causée par la mort du héros. Il n'empêche que les moments d'inhumanité l'emportent et le récit ne présente pas par hasard en alternance le premier concert d'Elias, dans la petite église, au jour de Pâques, avec l'effet quasi miraculeux de sa musique sur les grossiers fidèles, et une scène où Peter exerce sa vengeance sur un petit chat après avoir été sauvagement blessé par son père et avant de provoquer l'incendie du village (FS, pp. 69 sq.). Cette violence inhumaine est présente dès le début du texte avec le récit de la naissance du héros. Et la liste serait longue de toutes les scènes de sauvagerie qui émaillent le roman. L'épisode plaisant du 
charbonnier Michel et de son enthousiasme pour les Idées de Herder constitue d'une certaine manière un pendant à l'histoire d'Elias, lui aussi est à la recherche d'un idéal et croit pouvoir le trouver dans une Californie qu'il n'atteindra jamais. Il y a là chez lui aussi une étincelle positive, la nostalgie du vaste monde, l'aspiration moderne au savoir ; mais la fin du personnage est grotesque et mensongère. Les autres personnages sont davantage encore prisonniers d'une effrayante primitivité, en particulier les parents d'Elias, et même l'oncle Oskar, l'organiste et maître d'école, dont la semiculture envieuse et bornée ne lui permet pas de se libérer de traditions médiocres et asphyxiantes. Elsbeth est peut-être là, outre sa fonction sentimentale auprès du héros, pour que ne l'emporte pas en fin de compte la pure négativité. Certes, elle ne comprend guère celui qui lui voue un amour inavoué, absolu et fatal, mais elle ne participe pas pour autant de la simple médiocrité, voire de la barbarie générale. Elle est comme un personnage-témoin qui incarne une forme de normalité, elle qui, plus tard, épousera un médiocre, mais gardera, devant ses enfants, la mémoire de celui qui a traversé son existence et qu'elle n'a pu comprendre. Par elle, dans l'ultime chapitre du roman, l'histoire d'Elias prend déjà la coloration du mythe.

Ainsi, le roman entremêle les images négatives et positives, et cette ambivalence, qui est celle aussi de la nature et de Dieu, se retrouve tout particulièrement dans la figure du musicien Elias. En lui se rencontrent le pouvoir de la musique et l'échec du génie, une forme sublime de l'humanité et la monstruosité. Son destin est le parcours d'une révolte qui se retourne contre elle-même, il accuse Dieu de ne lui avoir donné le pouvoir de la musique que pour lui interdire l'amour. Or, à l'inverse du Nibelung, c'est l'amour qu'il choisit même s'il en sait la réalisation impossible et que cela le conduit à renoncer à la musique et à la vie elle-même. Ce Job, qui connaît une étrange nuit de révolte dans la solitude de la petite église où Dieu lui apparaît sous les espèces d'un enfant «sans nombril » (FS, p. 138), se résigne finalement même s'il sait que « Dieu est le plus fort car il aime toute injustice sous le soleil » (FS, p. 90). Peut-être le terme de grotesque est-il le mieux à même de caractériser ce monde ambivalent. Et d'une certaine manière, Elias est un antihéros grotesque, un mélange de génie et de monstre. Il ne manque pas de traits qui permettent de voir en lui une sorte de Christ grotesque : le visage monstrueusement métamorphosé de l'enfant semble "porter ensevelis tous les cris de douleur de l'homme et de la créature » (FS, p. 34) et Peter est une espèce de disciple, dévoué et effrayant à la fois, il est le seul à avoir entrevu le génie d'Elias et la disparition de son ami lui fera connaître une sorte de chemin de Damas. Enfin, l'étrange suicide d'Elias par privation de sommeil est clairement un acte de sacrifice au nom de l'amour ${ }^{12}$.

18 Pour notre propos, cette ambivalence fondamentale du monde de la fiction est importante dans la mesure où elle se retrouve dans l'image de la musique. Le génie musical est aussi une malédiction, le pouvoir de la musique est un sortilège démoniaque, mais aussi un flamboiement sans suite. Il n'est pas étonnant que cette problématique soit illustrée par une forme de musique qui, traditionnellement, est l'objet d'interprétations contradictoire, la musique d'orgue. On sait quel débat a suscité dans la pensée religieuse cet instrument dont la complexité et la puissance étaient bien faites pour impressionner les âmes, mais dont on pouvait craindre qu'il fût davantage la voix trompeuse du Malin que l'expression du Verbe divin.

19 Notons encore d'un mot, que les limites de cet article ne nous permettent pas de développer, les similitudes thématiques de notre roman avec un autre texte de 
R. Schneider, le roman Die Unberührten qui fait partie, on l'a dit, de la même trilogie. À travers le destin de la petite Antonia, l'équivalent d'Elias, on retrouve la même opposition entre bassesse et cruauté du réel, d'une part, et pouvoir de la musique, de l'autre. La voix joue ici le rôle de l'orgue, par elle Antonia exerce sur les êtres un pouvoir étrange et irrésistible de fascination.

Or, le fait que l'héroïne s'appelle Antonia nous conduit à un autre point de notre propos. Difficile en effet de ne pas rapprocher ce personnage de celui de Hoffmann dans la nouvelle Rat Krespel. La fille du conseiller possède également une voix d'une beauté surhumaine faite pour subjuguer tous ceux qui l'entendent. Mais l'idée de l'ambivalence du pouvoir de la musique était déjà présente, chez Hoffmann, à travers le motif de la maladie de la cantatrice: sa voix extraordinaire est le produit d'une monstruosité physiologique et chanter met en péril l'existence même de l'artiste, comme son père l'a compris. Antonia est finalement victime de ce pouvoir démonique de la musique lié, comme chez Elias, à celui de l'amour, puisque, malgré l'interdit paternel, elle finit par chanter pour celui qu'elle aime.

Avec sa constellation thématique autour de la musique, le roman de R. Schneider n'est donc pas un phénomène isolé et il est aisé de le rattacher à la tradition du Künstlerroman ou de la Künstlernovelle, comme bien d'autres récits de la littérature germanophone en particulier. Mais, le fait que l'histoire soit située dans les premières décennies du $\mathrm{XIX}^{\mathrm{e}}$ siècle est significatif à double titre si l'on considère la dimension intertextuelle de l'œuvre. D'abord, sur le plan musicologique, il est sans doute possible de voir une correspondance entre le génie musical d'Elias, sa manière nouvelle, virtuose, subjective et souveraine, de concevoir la musique d'église, et l'évolution de l'écriture et même des structures musicales au début du siècle. C'est un peu comme si Elias passait de la tradition de Bach à la musique de Liszt ou de Paganini. Il y a chez Hoffmann des pages sur la musique instrumentale dans lesquelles s'expriment à la fois l'admiration pour une musique moderne, libre et absolue, et une sorte de crainte devant cette puissance démonique que l'artiste ne maitrise peut-être plus.

Mais c'est sur le plan littéraire que l'intertextualité semble le plus riche. Nous avons déjà évoqué Hoffmann. C'est plus encore avec Wackenroder que les rapprochements sont intéressants. Ses essais, rédigés en collaboration avec Tieck, constituent, on le sait, une première réflexion romantique sur l'art, sur la peinture et la musique en particulier. Il serait trop long de citer tous les éléments du roman de R. Schneider qui ont leur équivalent dans ces textes, l'idée de la solitude du génie, du pouvoir de l'art sur les âmes, mais aussi celle de la nature étrange et inhumaine d'un destin qui coupe l'artiste du monde normal et le condamne à la souffrance. On sait que les dernières parties des deux recueils d'essais sont placées sous le signe de Joseph Berglinger, figure fictive de musicien dont Wackenroder retrace la destinée avant de citer des textes que Berglinger aurait lui-même rédigés. Berglinger, lui aussi, est un solitaire que les autres prennent volontiers pour un idiot, lui aussi a découvert la musique très tôt et seul, à travers l'expérience de la jouissance que lui procure la musique d'église. Lui non plus ne peut imaginer vivre pour autre chose que pour la musique et commence à composer sans connaître la moindre règle d'écriture. Surtout son destin, qui lui donne tout à la fois le pouvoir sur les âmes et la souffrance de la solitude, l'amène à s'interroger douloureusement sur le sens de son existence, à se tourner vers Dieu dans l'espoir d'une réponse ou pour lui lancer sa révolte ${ }^{13}$. Avant Kreisler, Berglinger est déjà l'artiste romantique qui souffre du philistinisme de la société et rêve d'un auditoire 
vibrant de la même ferveur que lui, de même qu'Elias compose une musique en isorythmie parfaite avec les battements du cœur de celle qu'il aime. Ce musicien qui meurt dans la fleur de l'âge après avoir composé une Passion était, dit le texte, « comme un homme malade qui, dans un étrange paroxysme, montre plus de forces qu'un être bien portant $»^{14}$. Il serait intéressant de montrer également la similitude de perspective entre la manière dont Wackenroder présente la biographie de Berglinger et celle dont le narrateur de R. Schneider commente la vie d'Elias. Ici et là le même ton personnel, la même sympathie et la même distance :

Pourquoi le Ciel voulut-il que durant toute sa vie la lutte entre son enthousiasme éthéré et la misérable bassesse de cette terre le rende tellement malheureux [...]?

Nous ne comprenons pas les voies du Ciel ${ }^{15}$.

Mais c'est dans les textes attribués à Berglinger qu'on trouve le plus clairement le thème de l'ambivalence de la musique et de son étrange proximité avec la folie et la monstruosité. Et notamment, le plus original et le plus proche du thème traité par R. Schneider, le «Conte oriental merveilleux de l'ermite nu ». Le lieu où vit l'ermite, une grotte près d'une rivière, annonce l'endroit où Elias vit sa métamorphose et sa mort. L'ermite, lui aussi, entend des sons que nul autre ne peut percevoir et cette ouïe surhumaine, qui le contraint à faire tourner sans cesse la « roue du temps » qu'il est seul à entendre, est pour lui aussi source de souffrance. Le pouvoir de la musique est un pouvoir tyrannique dans la mesure où celui qui en est habité ne peut percevoir le monde que sous le mode du rythme et de la mesure, et que cette nécessité intérieure l'isole du monde et lui interdit de vivre l'existence normale dont pourtant il rêve. Cependant, il faut noter une différence entre les deux textes. Dans la seconde partie du « conte » de Berglinger, l'ermite échappe à la souffrance de son existence justement par le pouvoir de la musique uni à celui de l'amour : il entend, par une splendide nuit d'été, " une musique céleste " provenant d'une barque qui emporte deux amants sur les eaux de la rivière. La musique semble être l'expression merveilleuse de leur amour et son pouvoir libérateur est si fort que l'ermite oublie à l'instant le rythme vide et obsédant auquel la vie pour lui jusque-là se réduisait, et comme dans un mouvement de danse, il est emporté au-dessus du monde terrestre et, au firmament, il devient une des étoiles de l'harmonie céleste. Les caravanes passant par là ont l'impression d'apercevoir au ciel «le génie de l'amour et de la musique ${ }^{16}$.

Cette dissemblance - plus dans l'idée que dans le style, au demeurant - nous amène à préciser notre rapprochement. Là où le conte romantique s'achève à la manière d'une légende sacrée, l'histoire d'Elias s'ouvre sur le vide et le paradoxe. Le génial musicien disparaît sans laisser de traces, sa mort est, quelque exalté que soit le sentiment qui la cause, un suicide. Et pourtant, Elias est un frère de Berglinger et le roman de R. Schneider une nouvelle variante du Künstlerroman. Simplement l'horizon du texte n'est plus l'idéalisme du xix ${ }^{e}$ siècle, les divers motifs et thèmes que le texte moderne entrecroise et agence (l'enfant prodige, le génie tragique, l'amour absolu, la musique et son pouvoir démonique etc.) sont l'expression d'une vision du monde où domine l'absurde ou l'arbitraire. La création littéraire elle-même (d'une certaine façon, le roman est construit de manière musicale, son écriture n'est pas sans ressembler parfois à la virtuosité du jeu d'orgue du héros) ne corrige que par sa vitalité paradoxale l'image d'un monde privé de sens. C'est pourquoi les thèmes traditionnels de l'idéalisme esthétique ou religieux sont ici en fin de compte tous marqués par le grotesque et par la distance du jeu souverain de l'écriture littéraire. Le pouvoir de la musique est à la fois un thème central du roman et il reflète cette ambivalence. Certes, R. Schneider, 
l'Autrichien, parle du pouvoir de la musique en des termes bien différents de ceux de sa compatriote Elfriede Jelinek dans La Pianiste: la musique n'est pas ici un monde qui concentre symboliquement et réellement les structures d'oppression sociale qui s'exercent sur l'individu, mais il serait inexact de prendre à la lettre ce que le roman emprunte à la tradition romantique. La musique d'Elias est géniale, mais elle ne laisse de traces ni sur les âmes des auditeurs qui n'en sont pas durablement marqués, ni dans le temps. Elle n'est en fin de compte qu'une pièce essentielle d'un jeu littéraire sans signification philosophique claire. Le paradoxe et l'absurde l'emportent, comme dans cette remarque du narrateur :

Aucun espoir n'a de sens. Que personne n'ait l'idée de vouloir que ses rêves s'accomplissent. On doit, bien au contraire, comprendre combien l'espoir est folie. Et lorsqu'on l'a compris, on peut alors espérer. Si l'on peut encore rêver, la vie a trouvé un sens ${ }^{17}$. (FS, p. 127)

Avec ce thème de la musique et de son pouvoir, R. Schneider poursuit une tradition importante de la littérature germanophone. Il le fait en tentant de pénétrer le mystère de la création et de la sensibilité musicales, comme, avant lui, l'avaient déjà réussi E.T.A. Hoffmann, Thomas Mann, Hermann Hesse ou, plus près de lui H.J. Ortheil. La virtuosité de l'écriture du roman, à l'image de la «magie » de la musique d'Elias, n'en fait ressortir que davantage l'image d'un monde "désenchanté ». C'est là la tonalité propre de cette fantaisie musicale.

\section{NOTES}

1. Die Luftgängerin, Munich, K. Blessing Verlag, 1998, p. 9.

2. Berlin, Aufbau Verlag, 2004.

3. «Rheintalische Trilogie », soit: Schlafes Bruder (Reclam-Verlag Leipzig, 1992), Die Luftgängerin (1998) et Die Unberührten (Munich, A. Knaus, 2000).

4. «Es ist eine Anklage wider Gott, dem es in seiner Verschwenderlaune gefallen hatte, die so wertvolle Gabe der Musik ausgerechnet über ein Eschberger Bauernkind auszugießen, wo er doch hätte absehen müssen, daß es sich und seine Anlage in dieser musiknotständigen Gegend niemals würden nutzen und vollenden können.» (SB, p.11) Par manque de place, nous ne pouvons insister comme il conviendrait sur l'importance et le rôle du narrateur auctorial dans l'économie du texte.

5. « Dann geschah das Wunder. An diesem Nachmittag hörte der fünfjährige Elias das Universum tönen » (SB, p. 32).

6. «Als er einmal träumend im Gras gelegen hatte, beobachtete er die Bahn zweier Zitronenfalter, wie sie fröhlich hin und her gaukelten. Und so fing er an, der alten Melodie [des Weihnachtslieds] eine neue Melodie hinzuzufügen.» (SB, p. 67)

7. «Er hatte die Menschen unter Hypnose gebracht. Sie saßen reglos in den Bänken, ihre Augenlider bewegten sich nicht mehr. Ihr Atmen hatte sich verlangsamt, und die Frequenz ihrer Herzschläge war die Frequenz seines Herzschlagens geworden. [...] In der unerschöpflichen Kombination von Akkorden herrschen nämlich Konstellationen, deren Erklingen im Hörer etwas entfesselt, was im Grunde nichts mehr mit Musik zu tun hat. » (SB, p. 176) 
8. «Gewaltig staunte das Kirchenvolk, als plötzlich beim Gloria die Orgel aufbrauste und mit jubelndem Fingerwerk anzeigte, auf welche Weise sich ein Christ über diesen Tag zu freuen habe. Elias spielte eine mächtig ausholende Toccata, die in einem fünfstimmigen Fugato über die Melodie des Kirchenliedes endete.» (SB, p. 112)

9. «Die Musik des Organisten machte ihre sturen Gemüter lammfromm, denn eigentümlicherweise verließ niemand die Kirche vor der Zeit. Es entstand auch nicht die leidliche Drängelei beim Weihwasserstock. Einige taten plötzlich ganz ungewöhnlich vornehm, gaben mit wurstigen Händen elegante Zeichen zum Vortritt und mengten in ihren Gruß - man wird es nicht glauben - Worte nach französischem Klang. » (SB, p. 113)

10. « Nein, der da oben machte nicht bloß Musik, er predigte.» (SB, p. 173)

11. «Sein ohnehin karges Gesicht war grau wir Asche geworden, die Wangen eingefallen, die Backenknochen standen heraus und die Lippen waren ihm vertrocknet. » (SB, p. 180)

12. Rappelons, pour souligner ce lien avec la figure christique, que le choral proposé à Elias et qui donne son titre au roman est le dernier mouvement de la cantate 56 de Bach dont le titre est : Ich will den Kreuzstab gerne tragen.

13. "Lieber Gott! ist denn das die Welt wie sie ist? Und ist es denn dein Wille, daß ich mich so unter das Gedränge des Haufens mischen und an dem gemeinen Elend Anteil nehmen soll ? (W. H. Wackenroder : Herzensergießungen, Klassiker Rororo, 1968, p. 94)

14. Ibid., p. 103 (trad. JChM).

15. Ibid.

16. « Ein wunderbares morgenländisches Märchen von einem nackten Heiligen », in ibid., pp. 152-155.

17. « Eine jede Hoffnung ist ohne Sinn. Kein Mensch verfalle auf die Idee, auf die Erfüllung seiner Träume zu sinnen. Vielmehr solle er den Irrsinn des Hoffens begreifen. Hat er ihn begriffen, darf er hoffen. Wenn er dann träumen kann, hat sein Leben Sinn. » (SB, p. 133)

\section{RÉSUMÉS}

Dans le roman de R. Schneider, le pouvoir de la musique apparaît d'abord à travers le destin du héros, Elias, jeune paysan d'un village perdu du Vorarlberg au début du xix ${ }^{\mathrm{e}}$ siècle. Le génie de la musique qui l'habite fait de lui un être hors du commun, à la fois monstrueux et sublime. À l'orgue, dont il apprend seul à jouer magistralement, il bouleverse toutes les règles connues et sa musique exerce sur les auditoires un pouvoir quasiment diabolique. Mais cette destinée est privée de sens dans la mesure où ce génie reste inconnu et disparaît sans laisser nulle trace. Le traitement du thème n'est pas sans évoquer la tradition romantique, en particulier la figure de Joseph Berglinger chez Wackenroder. Mais à l'opposé de cette tradition, et même si le thème se reflète dans la virtuosité de l'écriture, c'est ici l'absurde et le grotesque qui l'emportent.

Im Mittelpunkt von R. Schneiders Roman steht die Musik und ihre Macht, die zuerst im Werden der Hauptfigur verkörpert wird, des Elias, eines Bauernsohnes in einem verlorenen Bergdorf am Anfang des 19. Jahrhunderts. In ihm lebt der Genius der Musik, der ihn in ein halb monströses, halb sublimes Wesen verwandelt. Er lernt allein das Orgelspiel, indem er alle bekannten Regeln umwirft und so eine Musik schafft, die auf die Zuhörer mit dämonischer Kraft einwirkt. Jedoch ist das Schicksal dieses Genies sinnlos, weil es unbekannt bleibt und spurlos verschwindet. Die Art, wie der Roman das Thema der Musik verarbeitet, erinnert an vielen Stellen an die 
romantische Tradition, insbesondere ist Elias mit der Figur des Joseph Berglinger bei Wackenroder verwandt. Doch im Gegensatz zu dieser Tradition und obwohl sich das Thema nicht zuletzt in der Virtuosität des literarischen Schreibens widerspiegelt, ist die hier dargestellte Welt letzten Endes durch das Absurde und das Groteske geprägt.

\section{AUTEUR}

\section{JEAN-CHARLES MARGOTTON}

Université Lumière Lyon 2 